\title{
Developing and Testing Culturally Optimized Digital and mHealth Solutions for Hispanic, Multicultural, and International Audiences
}

\author{
Dirk Schroeder, ScD, MPH \\ Global Health, Rollins School of Public Health, Global Emory University \& HolaDoctor, Atlanta, GA, United States
}

\author{
Corresponding Author: \\ Dirk Schroeder, ScD, MPH \\ Global Health \\ Rollins School of Public Health \\ Global Emory University \& HolaDoctor \\ 196 Waverly Way NE \\ Atlanta, GA, 30307 \\ United States \\ Phone: 5204131 \\ Email: dschroeder@holadoctor.net
}

\begin{abstract}
Background: The vast majority of digital and mHealth applications are developed in English. In the United States, there are 52 million Hispanic people, over half of whom speak Spanish at home. In Latin America, there are nearly 400 million people online. To date, the majority of digital health solutions are either only in English or simply translated into Spanish or other languages, ignoring the cultural relevance and nuances of the target audiences. Culturally adapting digital health solutions may lead to increased engagement, utilization and effectiveness, but there have been few empirical studies comparing culturally adapted digital health solutions to simply translated ones.
\end{abstract}

Objective: 1) Compare the engagement and usage of culturally-adapted digital health content and programs that have been translated into Spanish with those that have been further culturally-adapted. 2) Generate guidelines for "best practices" for digital and mHealth developers seeking to engage US minority, multicultural and/or International audiences.

Methods: HolaDoctor built and operates the largest Spanish language health website on the internet, with 3 million unique visitors per month. In partnership with Consumer Reports Health, we tested translated versions of CR articles into Spanish with culturally-optimized versions that incorporated Hispanic imagery, examples, beliefs and Spanish-only phrases. In a separate study, we conducted a large randomized trial of over 1000 Hispanic health plan members in collaboration with three Blue Cross Blue Shield Plans, and compared a culturally-adapted health and wellness program with a bilingual, non-culturally adapted healthy weight program. The culturally adapted version was further tested and evaluated at scale in a state-wide Hispanic Obesity Prevention and Education (HOPE) program with the Florida Department of Chronic Disease.

Results: In comparison of a series of digital Consumer Reports articles, we found that the culturally adapted versions of the articles led to a $2 \mathrm{x}$ to $10 \mathrm{x}$ increased engagement and use than the translated only version. For example, with one article the translated only (TO) compared to the culturally adapted (CA) version received: 1499 (TO) vs 29,157 (CA) page views; an exit rate of $81 \%$ (TO) vs $20 \%$ (CA) and 3 (TO) vs 52 (CA) social shares. In the randomized trial, the culturally adapted version of the healthy weight program led to twice the engagement, twice the number of changes in positive healthy behaviors and a statistically significant in an intent to treat analysis greater weight loss 0.23 (TO) vs $0.52 \mathrm{lbs} /$ week; $P<.03$ ) over the 3 -month study period. In the Florida HOPE program, we found that cultural adaptation led to significantly increased consumption of fruits and vegetables and daily physical activity.

Conclusions: With now nearly $100 \%$ penetration of smart phones, digital health and mHealth solutions offer great promise for narrow the health disparities gap between the underserved multicultural populations and the general population. These solutions are also increasingly "going global." Simply translating digital and mHealth applications is better than English-only, but falls far short of the highly personalized, culturally-tailored solutions are capable of. Best practices for culturally-adapting digital health solutions at an affordable cost will be shared.

(iproc 2018;4(2):e11884) doi: $10.2196 / 11884$ 


\section{KEYWORDS}

cultural appropriateness; Hispanic/Latino; international resources

Edited by T Hale; this is a non-peer-reviewed article. Submitted 08.08.18; accepted 29.08.18; published 17.09.18.

Please cite as:

Schroeder D

Developing and Testing Culturally Optimized Digital and mHealth Solutions for Hispanic, Multicultural, and International Audiences iproc 2018;4(2):e11884

URL: http://www.iproc.org/2018/2/e11884/

doi: $\underline{10.2196 / 11884}$

PMID:

CDirk Schroeder. Originally published in Iproceedings (http://www.iproc.org), 17.09.2018. This is an open-access article distributed under the terms of the Creative Commons Attribution License (https://creativecommons.org/licenses/by/4.0/), which permits unrestricted use, distribution, and reproduction in any medium, provided the original work, first published in Iproceedings, is properly cited. The complete bibliographic information, a link to the original publication on http://www.iproc.org/, as well as this copyright and license information must be included. 